\title{
Integration of molecular biology tools for identifying promoters and genes abundantly expressed in flowers of Oncidium Gower Ramsey
}

\author{
Chen-Tran Hsu', De-Chih Liao', Fu-Hui Wu', Nien-Tze Liu', Shu-Chen Shen², Shu-Jen Chou ${ }^{3}$, Shu-Yun Tung ${ }^{4}$, \\ Chang-Hsien Yang ${ }^{5}$, Ming-Tsair Chan ${ }^{1,6^{*}+}$ and Choun-Sea Lin ${ }^{1 *+}$
}

\begin{abstract}
Background: Orchids comprise one of the largest families of flowering plants and generate commercially important flowers. However, model plants, such as Arabidopsis thaliana do not contain all plant genes, and agronomic and horticulturally important genera and species must be individually studied.

Results: Several molecular biology tools were used to isolate flower-specific gene promoters from Oncidium 'Gower Ramsey' (Onc. GR). A cDNA library of reproductive tissues was used to construct a microarray in order to compare gene expression in flowers and leaves. Five genes were highly expressed in flower tissues, and the subcellular locations of the corresponding proteins were identified using lip transient transformation with fluorescent protein-fusion constructs. BAC clones of the 5 genes, together with 7 previously published flower- and reproductive growth-specific genes in Onc. GR, were identified for cloning of their promoter regions. Interestingly, 3 of the 5 novel flower-abundant genes were putative trypsin inhibitor (TI) genes (OnT/1, OnT/2 and OnT/3), which were tandemly duplicated in the same BAC clone. Their promoters were identified using transient GUS reporter gene transformation and stable $A$. thaliana transformation analyses.
\end{abstract}

Conclusions: By combining cDNA microarray, BAC library, and bombardment assay techniques, we successfully identified flower-directed orchid genes and promoters.

\section{Background}

The Orchidaceae family comprises an estimated 35,000 species and is one of the largest families of flowering plants. The Oncidiinae subtribe consists of $\sim 70$ closely related genera and $>1400$ species, of which Oncidium is the largest genus $[1,2]$. Like other orchids, Oncidiinae can be easily crossed intergenerically, or across species, to produce flowers with unique colors, fragrances and shapes. Oncidium has become a commercially important flower in the orchid industry. Oncidium 'Gower Ramsey' (Onc. GR) is one of the most important Oncidium cutflower varieties; it is an interspecific hybrid derived from Onc. flexuosum, Onc. sphacelatum and Onc. varicosum. Onc. GR is a yellow flower variety that can flower year-

\footnotetext{
* Correspondence: mbmtchan@gate.sinica.edu.tw; cslin99@gate.sinica.edu.tw † Contributed equally

${ }^{1}$ Agricultural Biotechnology Research Center, Academia Sinica, Taipei, Taiwan Full list of author information is available at the end of the article
}

round. The length of inflorescence is $\sim 1 \mathrm{~m}$, with hundreds of ca. $4 \mathrm{~cm}$ flowers.

Functional genomic studies of orchids remain a challenge owing to large genome size, low transformation efficiency and long life cycles [3]. However, gene transformation of Onc. GR has been established [4], offering an alternative strategy for Oncidium breeding and making it a priority to investigate and obtain Oncidium promoters. To date, several strategies have been used to investigate orchids at the genomic level. Sequence homology searches have identified homologous genes in Oncidium [5-11], and expressed sequence tag (EST) databases have been used for gene cloning [12-18]. Because model plants, such as rice and A. thaliana, do not contain all plant genes, and because some genes related to the unique morphological and physiological characteristics of Oncidium, such as the flower and pseudobulbs cannot be identified using sequence homology, an Oncidium-specific cDNA library of pseudobulbs 
and flowers has been established that contains a large amount of genetic information [12-18]. However, gene expression patterns cannot be predicted by nucleic acid sequences. Furthermore, several of the non-model plant EST sequences are not full-length sequences.

To clone full-length genes and promoters, further processing is necessary, such as rapid amplification of complementary DNA ends (RACE) for full-length cDNA, or genomic walking for promoter studies $[8,15,16]$. These techniques are difficult to apply to Onc. GR because its genome is complex and has not been sequenced. Bacterial artificial chromosome (BAC) libraries are an alternative tool for full-length gene and promoter cloning. To obtain such libraries, genomic DNA is cut into pieces of $\sim 100 \mathrm{~kb}$, cloned into a vector and stored in bacteria, making it is easier to obtain the promoter and the full length of the target gene without interference from homologs in the genome. Various strategies can then be used to identify the clones that contain target genes [19-22], and the identified clones can be sequenced directly to obtain the full-length gene sequence.

In this report, a cDNA microarray, a BAC library and a bombardment assay were combined to establish a novel platform that was used to identify and clone the Onc. GR genes and promoters abundantly expressed in Onc. GR flowers. This approach, combining multiple tools provides a fast, easy to use and convenient strategy for obtaining useful genetic information about Oncidium.

\section{Results \\ Using cDNA microarray to identify genes highly expressed in flowers}

A cDNA microarray was used to identify genes that are abundantly expressed in flowers. PCR products of 1065 clones from the cDNA library of Onc. GR were spotted on to slides to establish a flower-derived microarray. A total of 77 clones were upregulated by $>3$-fold and 42 clones were downregulated $>3$-fold relative to the leaves (data not shown).

Sequencing revealed that several clones were repeated. Among the 77 clones corresponding to genes highly expressed in flowers, 57 were unique genes. Among the clones corresponding to genes highly expressed in leaves, 3 were related to photosynthesis/chloroplasts (chloroplast chlorophyll a/b-binding protein, NADH dehydrogenase, and photosystem II $10 \mathrm{kDa}$ protein) as expected; photosynthesis-related genes were highly expressed in leaves.

Genes in which the flower/leaf expression ratio was $>7.5$ are presented in Table 1. Gastrodianin and Aquaporin were duplicated in the microarray but appeared as different ratios. As no suitable RT-PCR primers for the gene similar to CAE01572.2 could be identified, RT-PCR of the
Table 1 Onc. Gower Ramsey genes that are abundantly expressed $(>7.5 \times)$ or repressed $(<0.06 x)$ in flower tissues

\begin{tabular}{|c|c|c|c|}
\hline Putative function & Clone ID & GenBank No. & $\mathrm{F} / \mathrm{L}$ \\
\hline \multicolumn{4}{|l|}{ Flower abundant } \\
\hline OnDRRP & $\mathrm{S} 1 \mathrm{H} 08$ & HS524704 & $22.86 \pm 9.50$ \\
\hline Cytosolic malate dehydrogenase & 08H08 & HS522502 & $16.81 \pm 10.64$ \\
\hline OnExpansin & $02 \mathrm{CO} 2$ & HS521943 & $14.59 \pm 8.26$ \\
\hline OnTI3 & $10 A 09$ & HS522609 & $10.85+4.89$ \\
\hline CAE01572.2_like & 06A05 & HS522251 & $10.17 \pm 4.44$ \\
\hline Gastrodianin-1 & S1G11 & HS524695 & $8.82 \pm 4.51$ \\
\hline Gastrodianin-2 & S1E09 & HS524669 & $8.77 \pm 4.97$ \\
\hline Aquaporin & 07D11 & HS522379 & $8.08+4.30$ \\
\hline OnTI1 & 03G05 & HS522068 & $8.07 \pm 4.76$ \\
\hline OnTI2 & S1D01 & HS524649 & $7.64+1.08$ \\
\hline \multicolumn{4}{|l|}{ Flower repression } \\
\hline $\begin{array}{l}\text { 3-phosphoinositide-dependent } \\
\text { protein kinase }\end{array}$ & 03D08 & HS522037 & $0.01 \pm 0.00$ \\
\hline Metallothionein & 07D07 & HS522375 & $0.01 \pm 0.00$ \\
\hline NP_085475.1 like & 09G06 & HS522583 & $0.02 \pm 0.01$ \\
\hline $\mathrm{NADH}$ dehydrogenase subunit & 06F02 & HS522306 & $0.02 \pm 0.01$ \\
\hline OnHy_O6B11 & $06 \mathrm{~B} 11$ & HS522268 & $0.02 \pm 0.02$ \\
\hline Chlorophyll a/b-binding protein & S1D02 & HS524650 & $0.03+0.02$ \\
\hline 405 ribosomal protein & 06D01 & HS522282 & $0.05 \pm 0.04$ \\
\hline OnHy_STA10 & $\mathrm{S} 1 \mathrm{~A} 10$ & HS524622 & $0.06 \pm 0.03$ \\
\hline
\end{tabular}

Values are presented as average \pm SD of 3 biological replicates $(n=3)$. "OnHy" denotes that no similar protein was identified using BlastX.

remaining 6 genes was performed to validate the microarray results. Cytosolic malate dehydrogenase was the only gene whose RT-PCR results were inconsistent with the microarray. The other 5 genes were highly expressed in reproductive tissues including flowers and stalks (Figure 1). Three of them, OnTI1, OnTI2, and OnTI3, shared sequence homology with known trypsin inhibitors (TI, Figure 2) and probably have similar functions. The remaining two, although highly expressed in flowers, were expressed at different development stages or in different flower organs (Figure 1). Disease resistance response protein $(O n D R R P)$ was expressed in fully blooming flowers and Expansin (OnExpansin) was highly expressed in the lip (labellum) extending stage. The 3 trypsin inhibitor genes were expressed at all stages, but most abundantly during the flower bud stage. In reproductive organs, OnExpansin and OnTI2 were predominantly expressed in the lips. OnTI3 was highly expressed in the callus.

\section{Promoter cloning using a BAC library}

Having used RT-PCR to confirm that these 5 genes were highly expressed in flowers, they were used for further promoter studies. BAC clones that contained the target genes were used for promoter cloning. There are $\sim 140,000$ clones in the Onc. GR BAC library. Because the target gene sequences were known, PCR was used 


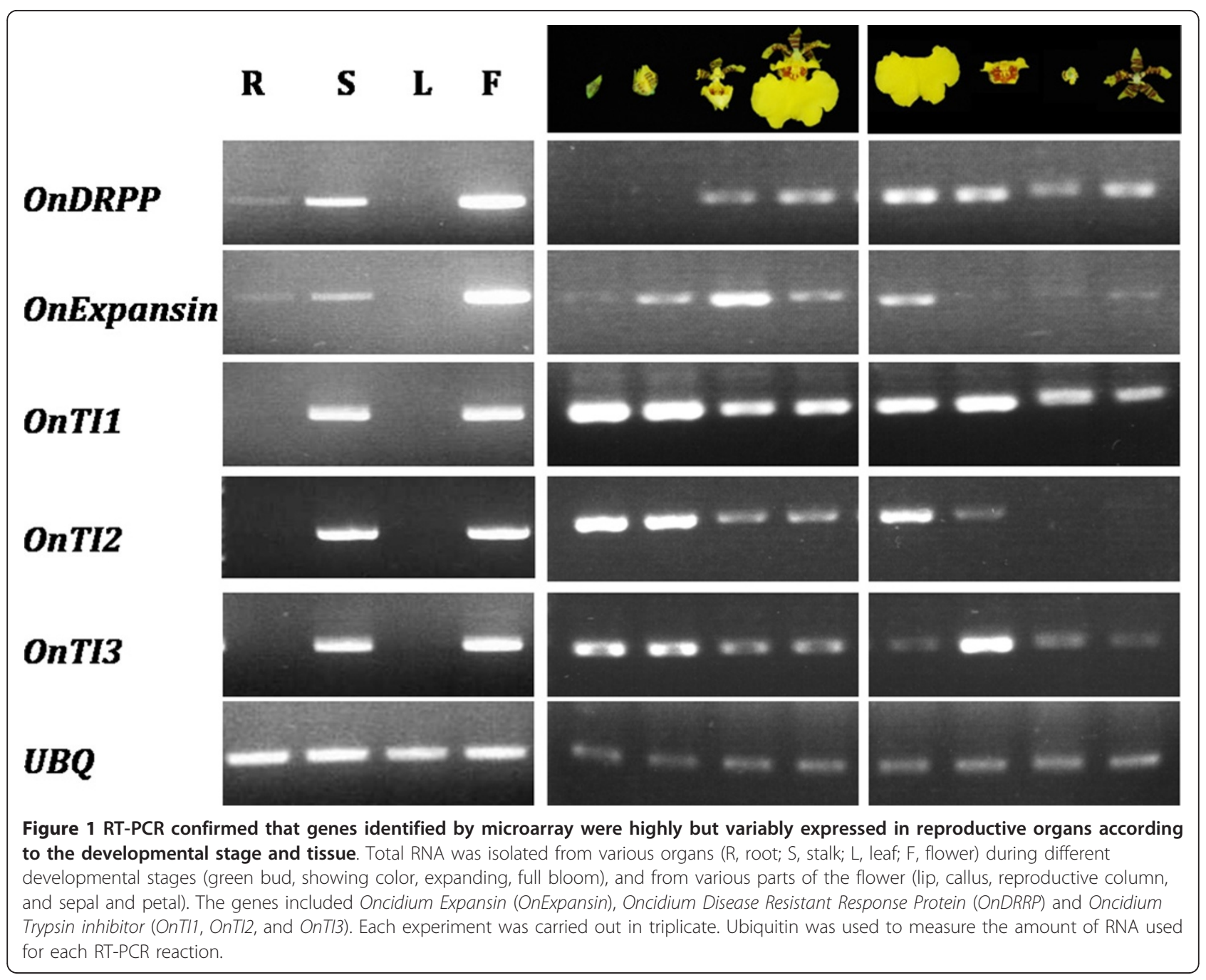

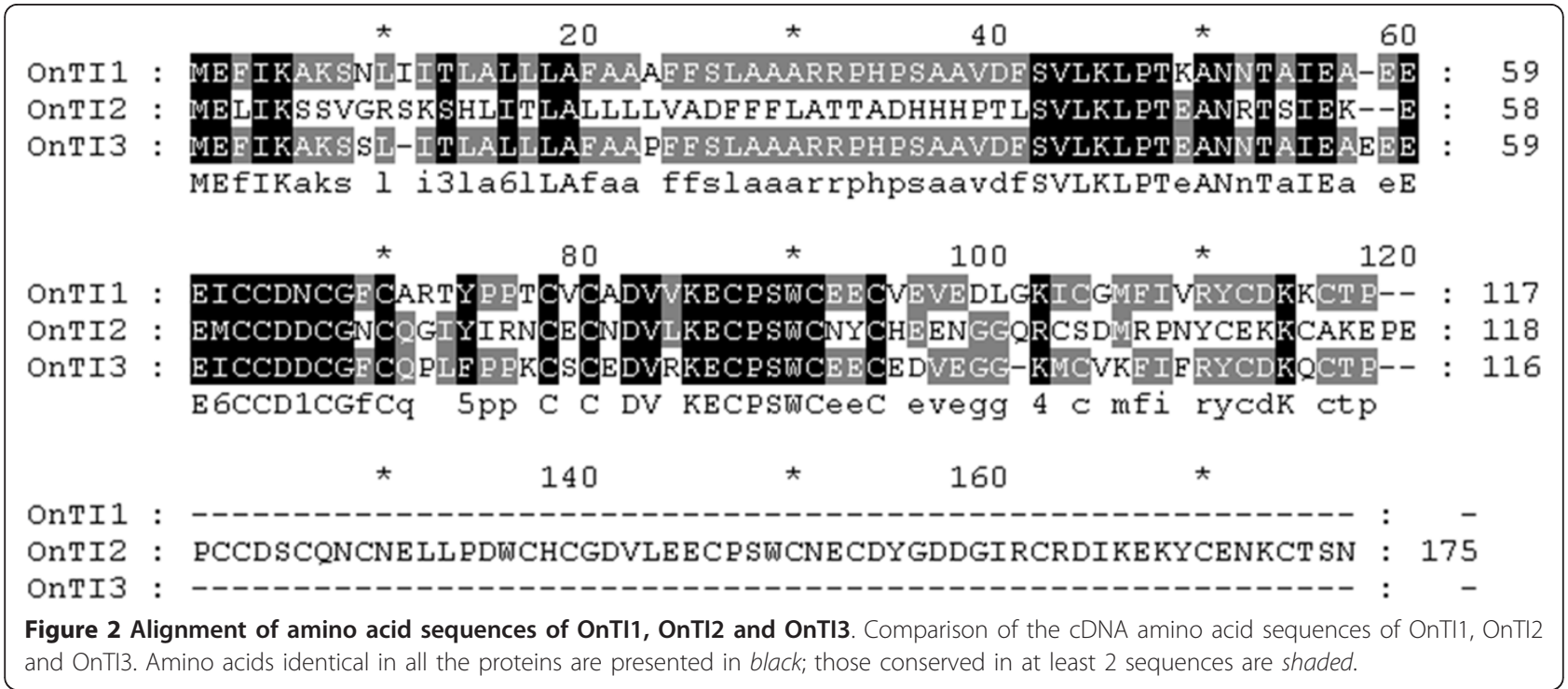


for screening. BAC screening was performed on a total of 12 genes; the 5 genes highly expressed in flowers as detailed above, and 7 previously published Oncidium flower-related genes (Table 2). These 12 genes were located in 10 different clones. Interestingly, the 3 trypsin inhibitor genes were located in the same clone, and tandemly duplicated sequences were found in OnTI2 and OnTI3. A hypothetical gene, OnHY1, was located between OnTI1 and OnTI2 (Figure 3). The putative protein sequence contains a Bowman-Birk serine protease inhibitor domain in the N-terminal region, similar to Lens culinaris trypsin inhibitor [GenBank: CAH04446.1]; and an amino acid sequence between 150 aa and 200 aa that is similar to a transposase domain.

\section{Identifying protein sub-cellular localization using fusion with fluorescent proteins}

Oncidium lip bombardment-mediated transformation was used to investigate the subcellular location of the protein products of the particular genes that were identified by microarray. Published protein markers were used to identify the organelles in the Oncidium cells of which the endomembrane system was most difficult to distinguish. Multiple protein markers derived from different plant species [23] indicated that these marker plasmids can be delivered into cells to synthesize fluorescent proteins (Figure 4A-E). Not only could the endomembrane systems be identified, but VirD2-NLS -mCherry (Figure 4F) could be used as a nuclear marker [24].

For the Oncidium genes investigated, no difference in the fluorescence patterns was observed when proteins were expressed as $\mathrm{N}$ - or $\mathrm{C}$-terminal fusions with a fluorescent protein (Figure 4G and 4H, OnTI1). The 3 OnTI proteins were seen as aggregated particles in the cells (Figure 4G-J). The subcellular locations of these proteins differed from endomembrane markers, such as mitochondria (Figure 4H). For YFP-OnExpasin, fluorescent signals were evident in the intercellular space and at the cell wall (Figure 4K), and for OnDRRP fluorescent signals appeared as a network system throughout the cell (Figure 4L).

\section{Use of multiple tools to identify promoters}

The 5 genes of interest were expressed in the lips; therefore, the Onc. GR lip was used for transient transformation. Oncidium alcohol acyl-transferase can be expressed in the leaves and flowers; its promoter (500 bp) was used as a positive control to demonstrate successful transformation. To investigate the promoter of OnTI1, various lengths (360, 740, 920, 1340, and $1913 \mathrm{bp}$ ) of the promoter region fused to the GUS reporter gene were introduced into the cells using the bombardment method. Plasmid pJD301 containing 35S-LUC was cobombarded as a reference control. The highest GUS activity was evident with the 920 bp length promoter. Interestingly, similar GUS activity was detected in the leaves using the leaves using the 360 and 740 bp lengths of the promoter region. GUS activities in the leaves were repressed in the transformants that had a promoter length of equal to or longer than $920 \mathrm{bp}$ (Figure 5). For OnExpansin, GUS activity in the leaves of all promoter transformants was low. GUS activity in the flower was correlated with promoter length, except for the $1027 \mathrm{bp}$ region, which had significantly reduced activity (Figure 6). Different lengths of OnExpansin promoter-GUS constructs were transformed into A. thaliana. With the exception of the 133 bp transformants, GUS activity was detected in flowers and minimal activity was present in the leaves (Figure 6). Various lengths of $O n T I 2$ and $O n D R R P$ promoters were constructed and a promoter assay was conducted (data not shown). The constructs

Table 2 Primers used for RT-PCR and BAC screening

\begin{tabular}{|c|c|c|c|c|}
\hline Gene & Forward primer & Reverse primer & Clone ID & GenBank No. \\
\hline UBQ & ACA TTC AGA AGG AGT CAA CCC & CGATGTCGATTTCGATTTCC & & \\
\hline OnDRRP & TGAAAAAGAAACCCATCTGCA & GCCCATAGGTGCCAATATTT & $P-5-0-22$ & HQ832781 \\
\hline OnExpansin & ACGCAACTITCTATGGCGG & AAGCAACCACAGCTCCAAGT & $0-1-0-24$ & HQ832782 \\
\hline OnTI1 & ATCACTTTGGCTCTGCTGCTT & TGCCGAGGTCCTCGACTTCCA & J-1-K-16 & HQ832783 \\
\hline OnTI2 & AAGAAGAACTCCCCACAAGAA & AGGTTGATCGATCGAAGCA & J-1-K-16 & HQ832783 \\
\hline OnTI3 & ATCACTTTGGCTCTGCTGCTT & AGCAATGAATGACGATCGAC & J-1-K-16 & HQ832783 \\
\hline OMADS3 & GAGGTATCAGCAAGTTACCG & CGAACGATCTTAATCGACTC & 45-3-B-1 & HQ832787 \\
\hline OMADS6 & AAACCCAGAGTAGTCAGCAG & GTCATATCCCATTGCATGA & $73-1-K-8$ & HQ832788 \\
\hline OMADS8 & ATGGAAGGCAGCATGAGAGAAC & AAAGCGTTAGCATTGTTACTTGTT & AAP-1-C-19 & HQ832789 \\
\hline OMADS9 & GATAAACCAAAACCTGAGGA & TITTGAAGGTATCGGTCTGG & $L-1-P-13$ & HQ832790 \\
\hline OnFT & ATTGTAGGACGAGTGATTGG & TACTTGGACTTGGAGCATCT & $Q-1-\mid-4$ & HQ832784 \\
\hline OnLeafy & TTCCTGGATCTCAACATCAT & TGCTGAAATCCTCAAACTTCA & Orp-2-F-21 & HQ832785 \\
\hline OnTFL & TTGTAGTTGGTAGAGTTATAGGAGAAG & ATCAGTCATAATCAGTGTGAAGAAAG & Q-1-B-10 & HQ832786 \\
\hline
\end{tabular}




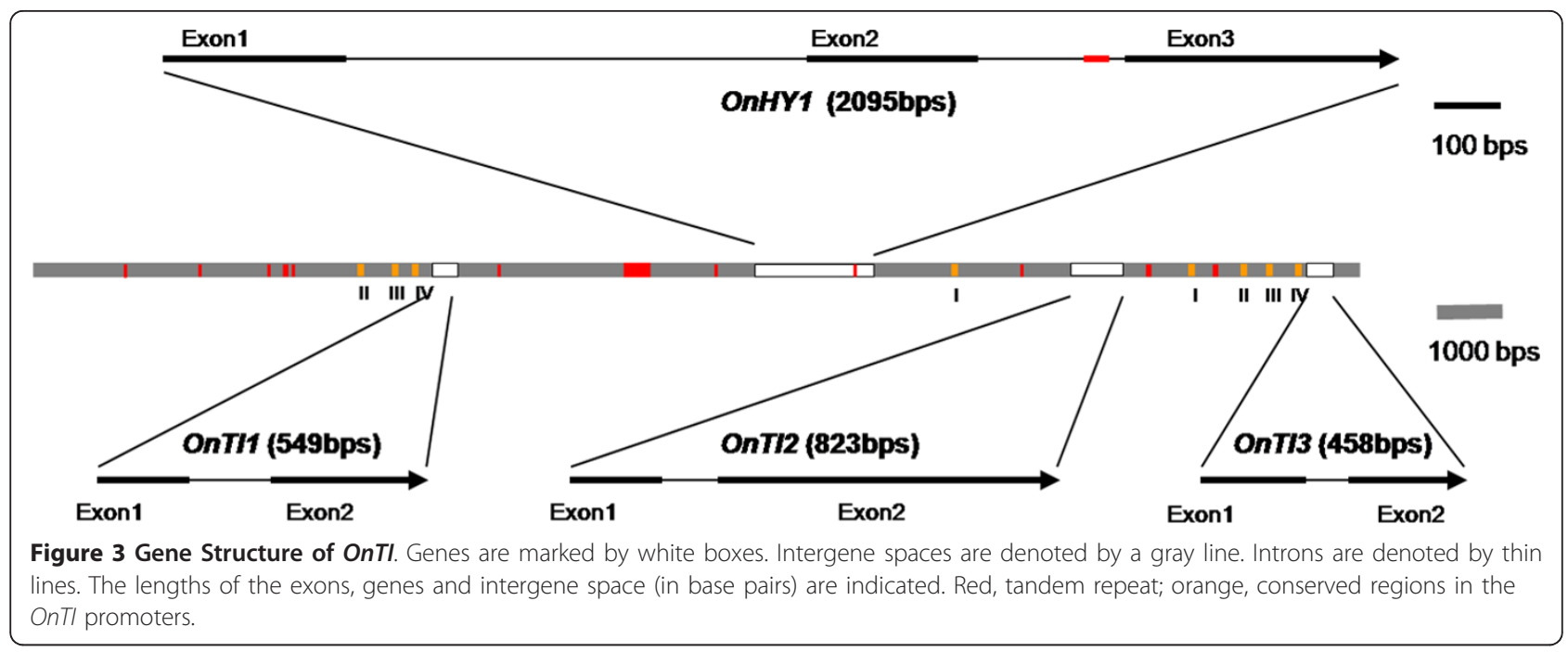

of OnExpansin, OnT1 and OnT2 yielding the highest flower/leaf GUS activity were then transformed into $A$. thaliana. The transformants of OnExpansin had the highest GUS activity in the flowers (Figure 6), whereas that of OnDRRP had the lowest (Figure 7). OnExpansin had GUS activity in the leaves (Figure 6). The flower GUS activity patterns for both OnTI1 and OnTI2 promoters were similar. Staining was observed at the top of the styles and at the junction of the pedicel and flowers (Figure 7).

\section{Discussion}

Identification of Oncidium reproductive-specific expression of genes using cDNA microarray

The aim of this study was to establish a successful combination of integrated tools to obtain genetic information about the commercially important cut flower Onc. GR. A combination of a cDNA library, a microarray, a BAC library and transient transformation was effective. However, the microarray and cDNA library that was used had several limitations: (1) In gene families that have conserved regions and share sequence identity, binding occurs that can limit the specificity of the data. For example, we found that gastrodianin, aquaporin and cytosolic malate dehydrogenase gave false positives. (2) The clone number was limited. There were only 1065 clones in the microarray, which cover only a fraction of the Oncidium genome. The estimated genome size is $1 \mathrm{C}=2.84 \mathrm{pg}$, http://data.kew. $\mathrm{org} /$ cvalues/CvalServlet?querytype $=1$. The estimated coverage of the Onc. GR BAC library is thus 1.28 fold, thereby limiting its possible uses. (3) Only a few genes that are highly expressed in leaves were identified because the microarray was composed from a flower cDNA library. To widen the use of this array, more sequence information needs to be integrated. For example, further libraries must be derived from different tissues and treatments.
Sequences from next generation sequencing are an alternative resource for obtaining this data. In comparison to the traditionally employed method (i.e. construction of an EST library, storage and sequencing of each clone using Sanger sequencing technology), using high-throughput approaches allows several thousand ESTs to be obtained cost-effectively from different tissues with less space and effort. Specific gene sequences can then be printed and a microarray yielding more detailed data can be useful for a variety of applications.

\section{BAC library construction is a useful tool for cloning promoters}

Polyploidy is a common phenomenon in crop species. In the indigenous species of Oncidium, the chromosome number is $2 n=56 \mathrm{http} / /$ data.kew.org/cvalues/CvalServlet?querytype $=1$; however, the chromosome number in Onc. GR is 112. Therefore, it is expected that there are several homologous genes in the genome of Onc. GR. In addition, tandem duplication, such as that found in the OnTI genes, or tandem repeat sequences such as those found in OnFT and OMADS9, would render genome walking using a PCR strategy particularly difficult to perform (Table 3). In many cases, it would take several months to identify a single gene. By screening a BAC library, target genes are narrowed down to those with lengths of $100 \mathrm{~kb}$, thereby reducing the problems related to homologous genes, tandem repeat sequences and secondary structure. In addition, the PCR strategy used herein can identify the BAC clone containing a target gene within a week, and regions of interest can be sequenced using BAC End Sequencing (BES).

Two strategies are used for BAC library screening: hybridization and PCR screening. As the gene sequences of the target genes were known in this study, the PCR screening strategy could be adopted. Recent improvements in PCR 


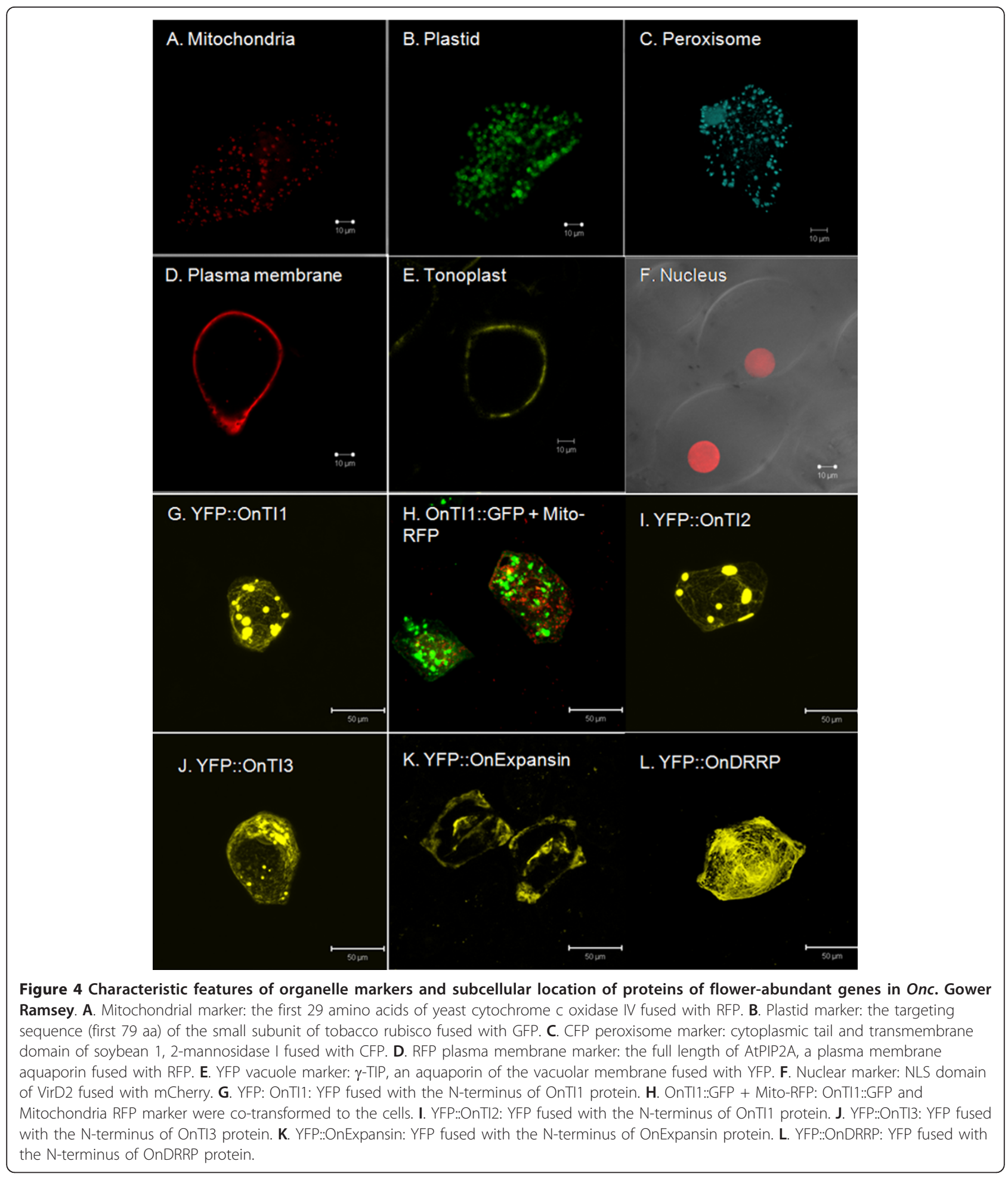

technology and protocols have made BAC screening more efficient and several genes have been successfully cloned using PCR to screen BAC libraries [19-22]. We thus used this strategy to obtain BAC clones containing genes of interest in the Onc. GR library.
Three trypsin inhibitor genes, OnTI1, OnTI2 and OnT/3, which are highly expressed in flowers, are tandemly duplicated

Three tandemly duplicated genes, OnTI1, OnTI2 and OnTI that are highly expressed in flowers were 


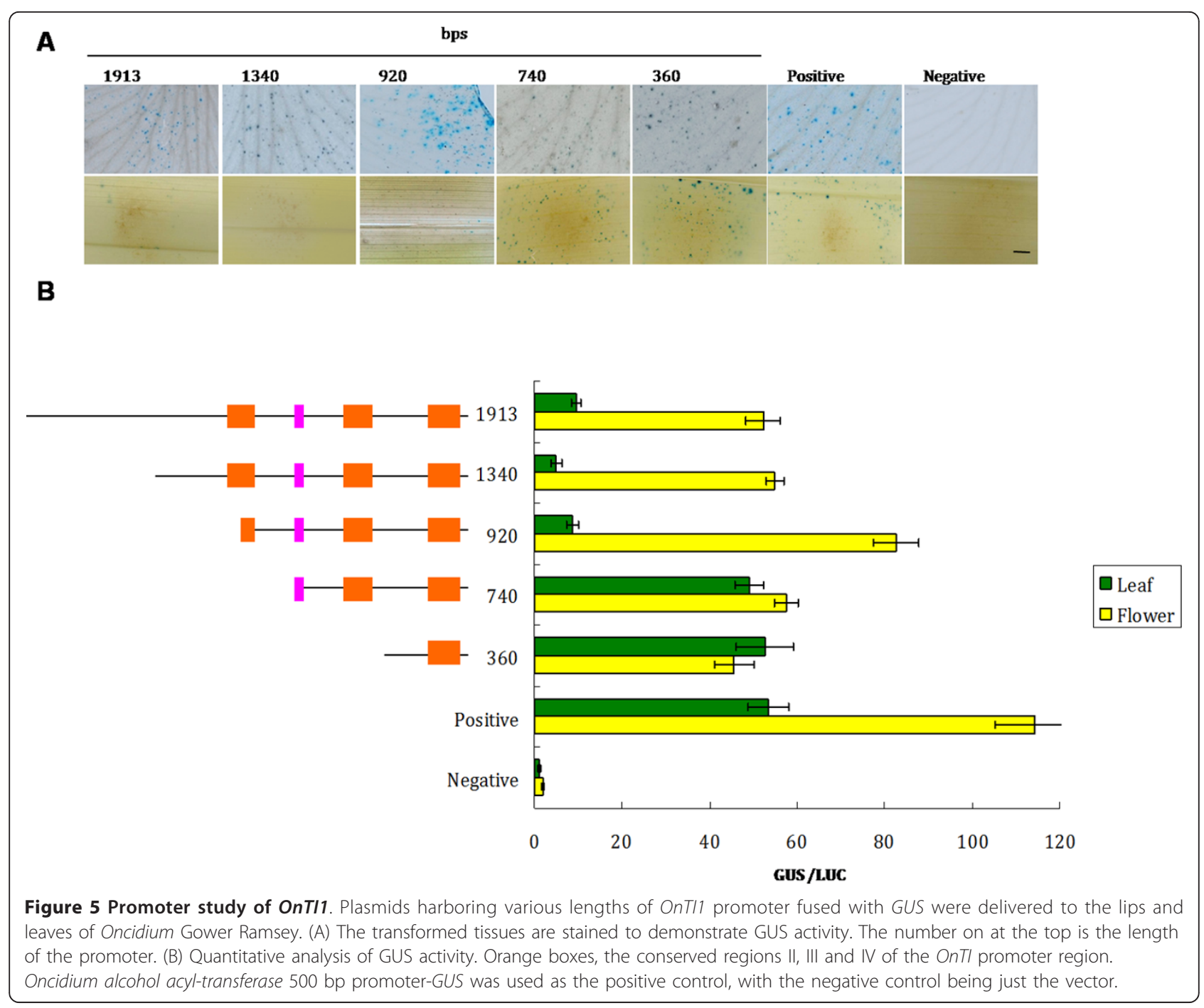

identified. Gene duplications that encode similar gene functions are a common phenomenon in plants and are thought to have contributed to the origin of evolutionary 'novelties' [25]. For example, it has been proposed that in the early evolution of orchids, two rounds of DEFICENS-like MADS-box gene duplications generated the genes that were probably recruited to distinguish the different types of orchid perianth organs [25]. Information about tandem duplicates can be useful in investigations pertaining to gene duplication. For example, the cinnamyl alcohol dehydrogenase gene [26], the R2R3$M Y B$ family of transcription factors genes [27] and NAC domain transcription factors genes [28] are tandemly duplicated in Populus trichocarpa. These genes have been duplicated from the same ancestral gene, allowing the expression pattern of these genes to be correlated. An investigation of the gene locations of the NAC domain transcription factors in Populus trichocarpa showed that 6 pairs of NACs are present as tandem duplicates, represented in tandem clusters of 2 or 3 genes each. In the tandemly duplicated clusters with 3 genes, the expression patterns of 2 of the genes were found almost identical. However, in the tandemly duplicated clusters with 2 genes, the gene expression levels differed significantly [28]. In the current study, the expression patterns of OnTI genes were similar. On the basis of sequence homology, we discovered 4 conserved regions upstream of OnTI3 similar to OnTI2 (region 1) and OnTI1 (regions 2-4). We tentatively suggest that these OnTIs may be derived from the same ancestral gene.

Several di- or tri-nucleotide tandem repeats were evident in the flower-related genes (Table 3). Because information on Oncidium is limited, the biological significance of tandem repeats in these genes remains unclear. The end sequencing of this BAC library may 


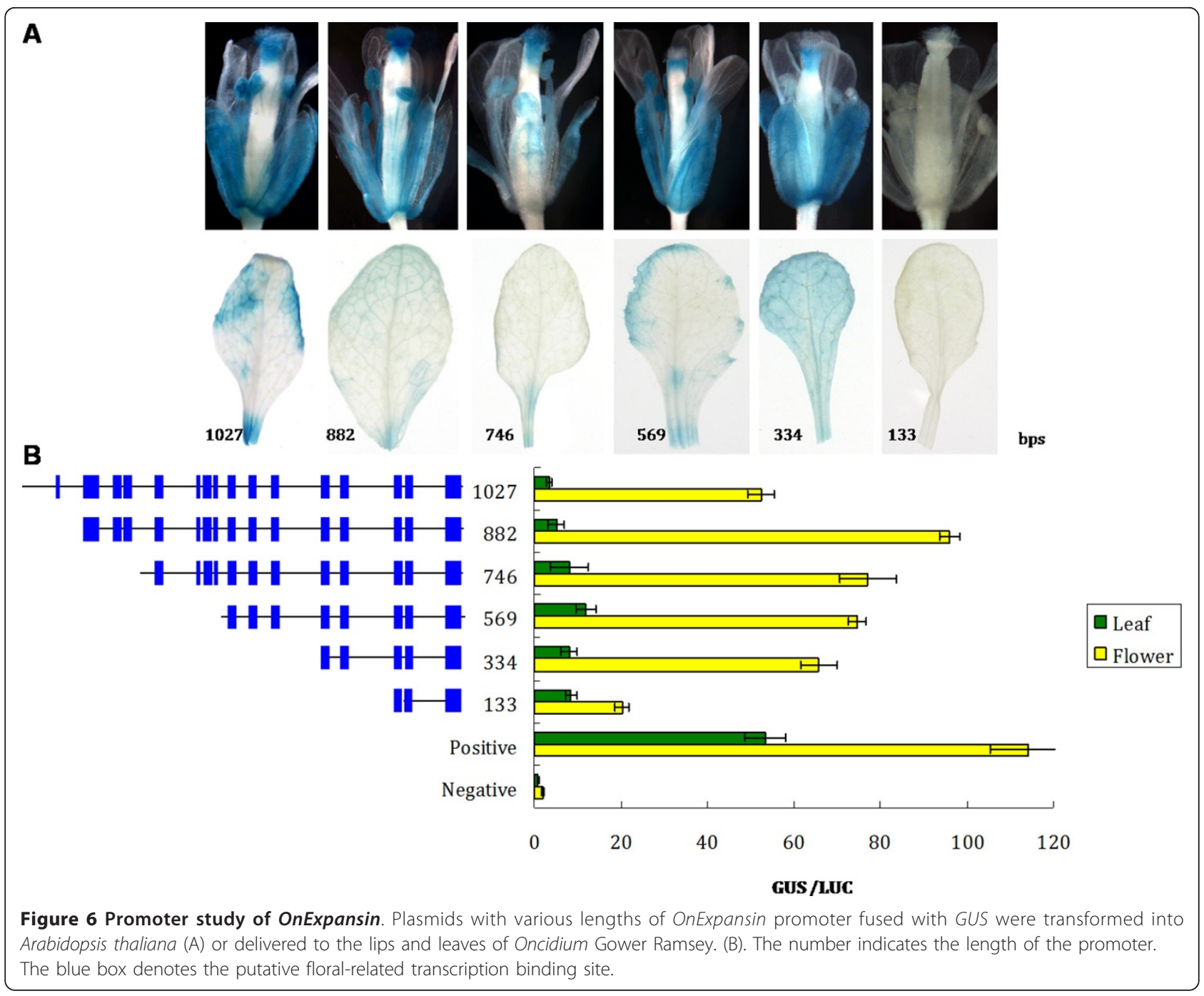

provide suitable information for identifying the relationship between flower-related genes and tandem repeat sequences.

\section{Transient transformation is a suitable tool for} determining the subcellular localization of protein The subcellular location of a protein is related to its function. For example, photosynthesis-related proteins are located in chloroplasts. Therefore, experiments aimed at determining the specific localization of proteins can provide information on biological processes [29]. Computational prediction is one method used to investigate the subcellular localization of a protein [29]. However, as yet, no suitable reference database exists for Oncidium. Experimentally, the subcellular localization of a protein can be studied by imaging it after fusion with a fluorescent protein [30,31]. However, no suitable protocol for investigating subcellular localization has so far been established for orchids. In this report, a transient transformation system for the orchid lip using markers derived from different species as fluorescent markers was established to study subcellular localization of proteins.

Trypsin inhibitors can be used to reduce trypsin activity, which can play an active role against pests and diseases [32]. The expression of trypsin inhibitor genes can also be induced by water stress [33] and stress-related plant growth regulators [34,35]. Constitutive expression of a trypsin inhibitor can improve plant tolerance to abiotic stress $[34,35]$. Trypsin inhibitors are present in all protein bodies, and to a lesser extent in the nucleus and intercellular space [36,37]. Here, we found that OnTI proteins can form particles similar to protein bodies, but they were not in the nucleus or intercellular space.

Expansins are a superfamily of proteins crucial in loosening the cell wall. The expansins consist of 2 domains, the glycoside hydrolase family 45 (GH45) 




Table 3 Tandem repeats in the promoter and gene sequences used in this report.

\begin{tabular}{llll}
\hline Gene & Position & Repeat & Copies \\
\hline OnExpansin & -1478 & AATAAA & 33 \\
OnTI1 & -3692 & A & 34 \\
& -2047 & CT & 14.5 \\
OnTI2 & -7766 & TTA & 167 \\
& -6130 & TA & 26 \\
OnTI3 & -2662 & TTA & 23.7 \\
& -1489 & AAT & 30 \\
OMADS3 & -1003 & TAT & 56.7 \\
OMADS6 & -1234 & ATA & 13.3 \\
OMADS9 & 1079 & A & 26 \\
OnFT & -66 & CTT & 8.7 \\
OnLeafy & -1167 & TAA & 25 \\
\hline
\end{tabular}

catalytic domain and group-2 grass pollen allergens. Experimental evidence indicates that expansins can induce slippage of cellulose microfibrils in the cell wall which becomes loosened [38]. The expansin was located in the cell wall and in the intercell wall spaces $[39,40]$. The fluorescent signal for OnExpansin was located around the cell wall; according to the results obtained using RT-PCR, OnExpansin was highly expressed in the lips and during lip expansion. Therefore, this gene may be correlated with Onc. GR lip development.

In summary, the localizations of the proteins we investigated are correlated with their predicted functions, but the roles of these genes during Oncidium flower development are unknown as their overexpression in A. thaliana flowers did not result in any significant change in terms of flowering time or morphology. 


\section{Useful genetic information can be mined using this integrated platform}

Promoters of Oncidium were successfully cloned using a combination of a cDNA library, microarray, BAC library and transient transformation. Transformation of Oncidium is time-consuming and requires considerable human resources. Use of a transient expression system reduced the time required to obtain preliminary information to $\sim 1$ week. This approach is thus more timeefficient than genomic walking and stable transformation methods, and allows investigators to estimate experimental priorities.

There are 4 conserved regions in the promoter regions of OnTI genes. The OnTI1 promoter study demonstrated that box 1 , box 3 and box 4 were not related to flower expression. The OnTI2 promoter, which does not have these regions, can be expressed in flowers. The most important region controlling the repression in leaves is situated between box 2 and the repeat region. There is a potential Agamous binding site in this region and there is a similar region in the OnTI2 promoter region (TAATGTTACGAAATAAAATATCACTCCTGAATATA). Unlike the repression of OnTI2 in leaves, the most important region for flower expression in OnExpansin is located between -113 to $-334 \mathrm{bp}$. It is expected that the regulation of OnExpansin expression is different from that of OnTI2. Interestingly, 2 potential TF-binding domains (an Agamous and an AtHB9 binding site) are flower or development related. The relevance of the Agamous binding site for gene repression in leaves and flower expression, however, requires further investigation.

The promoter regions of OnTI, OnExpansin and Oncidium $M A D S$ genes contain nucleotide tandem repeat sequences (Table 3). However, promoter studies demonstrated that the tandem repeats in OnTI1 and OnExpansin promoters are not related to gene expression. According to our data, the promoter region controlling flower/leaf expression is within $1 \mathrm{~kb}$ of the promoter. Analysis of other gene promoters (OnTI1 and OnDRRP) produced similar results (data not shown).

The clones which contain $\sim 1 \mathrm{~kb}$ promoter regions fused with GUS were transformed into A. thaliana. Although GUS staining was more prominent in flowers, there were some unexpected results. In OnExpansin, GUS staining was evident in the leaves despite the RTPCR results demonstrating that OnExpansin is predominantly expressed in the lips of Oncidium. In A. thaliana, GUS was weakly expressed in petals, but highly expressed in anthers and styles (Figure 6). The OnTI genes were predominantly expressed in the Oncidium lip and callus. However, there was no GUS staining in the petals of the A. thaliana transformants. These results may be due to the absence of a transcription factor that can recognize the Oncidium binding site, highlighting the necessity of identifying species-specific promoters. The promoters we found were only $1 \mathrm{~kb}$ in size. The region that controls the specific organ of interest may not have been included, producing unexpected results in stable $A$. thaliana transformation.

\section{Conclusions}

A cDNA library, a microarray, a BAC library and transient transformation were combined to identify gene promoters highly expressed in the flowers of Oncidium Gower Ramsey, a commercially important cut flower. Classical approaches of identifying orchid genes and promoters - in particular the genome walking method - cannot easily be performed when regions of high DNA sequence homology tandem repeats and tandemly duplicated genes are present. Gene sequences of interest were identified successfully using BAC sequencing. Using lip transient transformation, GUS reporter gene fusion constructs with various lengths of promoters were introduced into the lip to determine promoter activity. Furthermore, the subcellular localization of proteins encoded in these genes was also determined in this system. With this combination of approaches, 5 novel Oncidium gene promoters of genes abundantly expressed in flowers were cloned and confirmed. These promoters can be used to express genes in floral organs and change the flower phenotype without affecting the vegetative tissues.

\section{Methods}

\section{Plant materials}

Flowering Onc. GR (a tetraploid interspecific hybrid) were obtained from a local grower (Yung Hsin Orchid Nursery, Taichung, Taiwan). The orchids were maintained in the greenhouse at Academia Sinica, Taipei, Taiwan. A voucher specimen was deposited at the National Museum of Natural Science, Taichung, Taiwan.

\section{Onc. Gower Ramsey flower cDNA library construction}

Onc. GR flowers were used as the materials for cDNA library construction. Total RNA and poly(A)+ mRNA were isolated using Trizol reagent (Invitrogen, Carlsbed, CA, USA) and the Oligotex Midi mRNA kit (Qiagen, Venlo, The Netherlands), respectively, according to the manufacturer's instructions. The cDNA library was constructed using the Long Distance PCR SMART cDNA Library Construction kit (Clontech, Mountain View, CA, USA) following the manufacturer's instructions. The cDNAs were cloned into the pDNR-LIB vector (Clontech). Colonies were picked up, collected in 96-well plates, and stored at $-80^{\circ} \mathrm{C}$. 


\section{Microarray preparation}

Microarray preparation followed the procedure described by $\mathrm{Wu}$ et al. [41] for the preparation of a bamboo microarray. A total of 1065 cDNAs [GenBank: HS521830-HS522791; HS524614-HS524707] derived from the Onc. GR flower cDNA library were amplified using PCR, incorporating the T3 and M13 reverse universal primers. The PCR products were purified using the MultiScreen PCR $_{96}$ Filter Plate (Millipore Corp., Bedford, MA, USA) and eluted with $100 \mu \mathrm{l}$ of $0.1 \times \mathrm{TE}$ buffer (1 mM Tris and 0.1 mM EDTA, pH 8.0). Purified PCR products were printed on GAPS II-coated slides (Corning, New York, NY) using the OmniGrid 100 microarray (Genomic Solutions, Ann Arbor, MI, USA), and arranged into two $1.8 \times 1.8-\mathrm{cm}$ arrays (spot size: $100 \mu \mathrm{m})$. After printing, the slides were left to dry overnight. These DNAs were cross-linked to the slide by baking the array for $2 \mathrm{~h}$ at $80^{\circ} \mathrm{C}$.

Total RNA from leaves and flowers $(25 \mu \mathrm{g})$ was used for cDNA synthesis and labeling with either Cy3 or Cy5 dye, using the 3DNA Expression Array Detection kit for microarrays (Array 50, version 2, Genisphere, Hatfield, PA, USA). cDNA hybridization and washing procedures were performed according to the manufacturer's instructions. All experiments were carried out in 3 biological replicates $(n=3)$. Detailed information of the microarray has been deposited in the NCBI GEO database [GEO: GSE26504].

\section{Semi-quantification using RT-PCR}

Total RNA $(5 \mu \mathrm{g})$ extracted from various tissues was subjected to RT-PCR. First-strand cDNAs were synthesized using M-MLV reverse transcriptase (RNase H Minus, Point mutant; Promega, Madison, WI, USA) and a poly (dT) primer. Each gene was amplified for 25 cycles using primers specific for each gene. Onc. GR ubiquitin was used as an internal control. The primers are given in Table 2.

\section{BAC library construction}

Young Onc. GR leaves (200 g) were collected for isolation of high molecular weight DNA according to Zhang et al. [42]. The DNA was sheared randomly, and the fragments ligated into the pSMART-BAC vector (Lucigen, Middleton, WI, USA). The ligated DNA was transfected into $E$. coli strain 10G BAC-Optimized Electrocompetent cells (Lucigen).

\section{Identification of BAC clones containing target genes using PCR}

The BAC library of Onc. GR DNA in E. coli was spread on solid medium plates $(23 \times 23 \mathrm{~cm})$ containing Luria broth (LB) supplemented with $25 \mathrm{mg} / \mathrm{L}$ chloramphenicol. Each plate contained $\sim 1000$ clones. After overnight incubation at $37^{\circ} \mathrm{C}$, individual clones were picked up by a Q-bot robot (Genetix, New Milton, UK) and placed into a 384-well plate that contained liquid LB medium with $12.5 \mathrm{mg} / \mathrm{L}$ chloramphenicol. Clones the robot failed to identify were picked up manually. The 384-well plates were incubated at $37^{\circ} \mathrm{C}$ overnight and stored at $-80^{\circ} \mathrm{C}$. The plates were washed with LB liquid medium containing $12.5 \mathrm{mg} / \mathrm{L}$ chloramphenicol, incubated overnight at $37^{\circ} \mathrm{C}$ and stored at $-80^{\circ} \mathrm{C}$ for the superpool (Figure 8).

Sequences derived from the microarray experiments and the published flower-related genes were used to design primers. Primers that could amplify predicted genomic regions in the presence of Onc. GR genomic DNA (positive control, white box, Figure 8) were used for further screening. After superpool screening (149 reactions), plate screening (2-5 reactions) was performed, and row (16 reactions) and spot screening (24 reactions) were used to identify clones containing genes of interest.

\section{BAC plasmid isolation and sequencing}

BAC plasmids were isolated using the NucleoBond BAC 100 kit (NucleoSpin blood, Macherey-Nagel GmbH \& Co KG, Germany) following the manufacturer's instructions, and sequenced using the Big Dye ${ }^{\mathrm{TM}}$ Terminator Cycle Sequencing Ready Reaction kit and an automated sequencer (Perkin-Elmer Applied Biosystems, CA, USA).

\section{Transient transformation}

Bombardment assay was conducted as outlined below, modified from Chiou et al. [14]. Purified recombinant plasmid DNA $(2.5 \mu \mathrm{g})$ was isolated using the Midi Plus plasmid DNA extraction system (Viogene, Taipei, Taiwan) and coated onto gold particles (1 $\mu \mathrm{m}$ diameter) for bombardment transformation. Onc. GR flower lips and leaves were incubated on sucrose-free 1/2 MS [43] solid medium and bombarded using a pneumatic particle gun (Biolistic PDS-1000/He; Bio-Rad) set to the following conditions: 1350 psi helium pressure of projectile; 27 $\mathrm{mm} \mathrm{Hg}$ partial vacuum; $9 \mathrm{~cm}$ target-distance. Bombarded lips were subsequently incubated on MS solid medium at $22^{\circ} \mathrm{C}$ overnight for further experiments.

\section{Subcellular localization}

Full-length cDNAs were amplified using PCR incorporating Onc. GR flower cDNA as template. The primer information is listed in Table 4. Products were cloned into pDONR221 by Gateway BP Clonase II Enzyme Mix (Invitrogen), and into p2YGWF (cGFP) and p2GWF7 (N-YFP) using Gateway LR Clonase II Enzyme Mix (Invitrogen) [44]. The plasmids were isolated and transformed into the lips using bombardment transformation. The transformed lips were observed on Zeiss LSM 510 META laser-scanning confocal microscope using an LD C-Apochromat $40 \times / 1.1 \mathrm{~W}$ objective lens. Excitation wavelengths and 


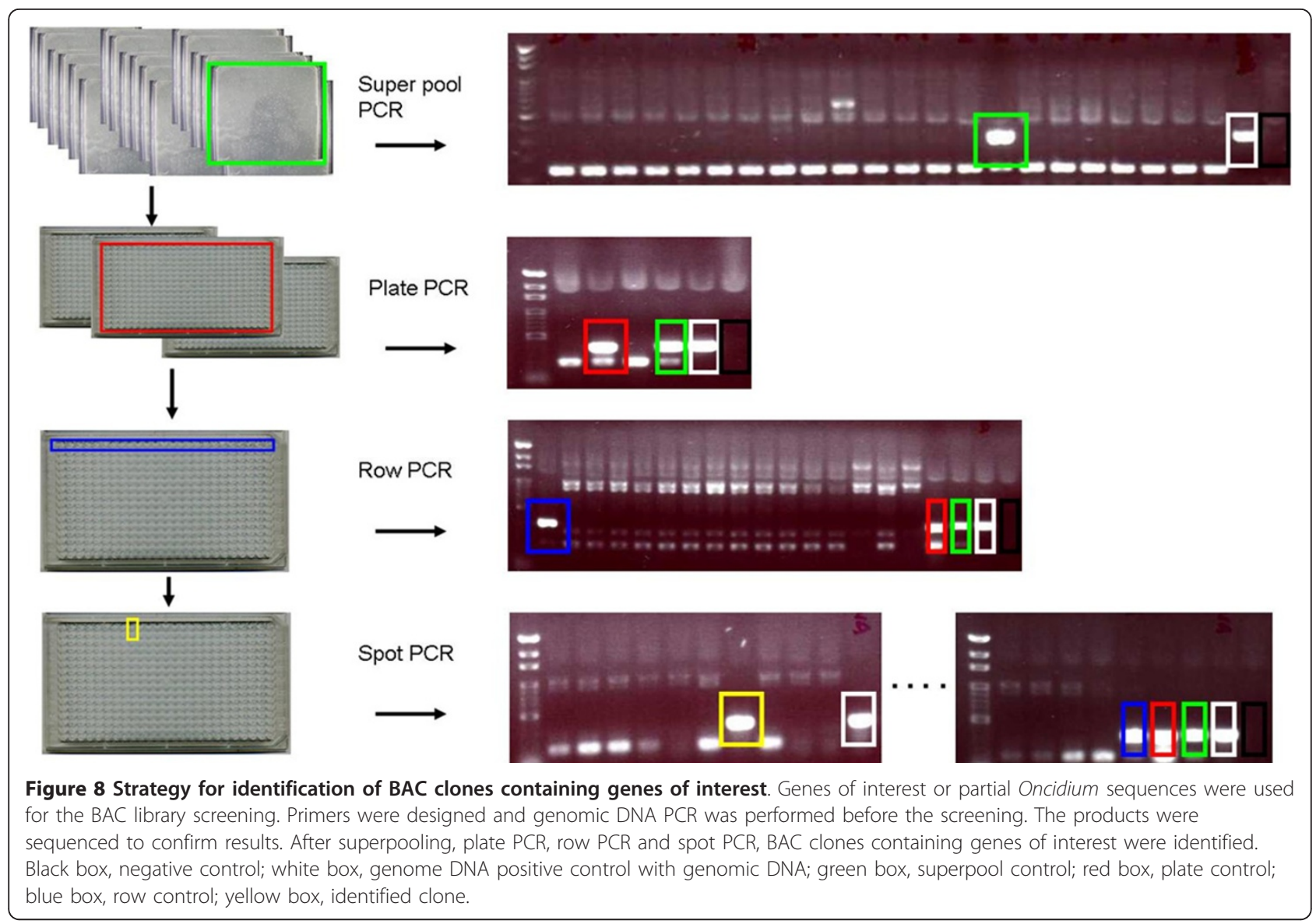

emission filters were $458 \mathrm{~nm} /$ band-pass $465-510 \mathrm{~nm}$ for CFP, $488 \mathrm{~nm} /$ bandpass $500-530 \mathrm{~nm}$ for GFP, $514 \mathrm{~nm} /$ bandpass $525-555 \mathrm{~nm}$ for YFP, $561 \mathrm{~nm} /$ bandpass 575-630 $\mathrm{nm}$ for RFP and mCherry, and $488 \mathrm{~nm} /$ band-pass $650-710$

Table 4 Primers used for the construction of fluorescence protein fusion

\begin{tabular}{|c|c|c|}
\hline Gene & $\begin{array}{l}\text { Primer } \\
\text { No. }\end{array}$ & Sequence \\
\hline \multicolumn{3}{|l|}{ GFP } \\
\hline \multirow[t]{2}{*}{ OnTI1 } & 1894 & AAAAAGCAGGCTTCATGGAGTTCATCAAAGCAAAG \\
\hline & 2129 & AGA AAGCTGGGTCAGGAGTACACTITTAT \\
\hline \multicolumn{3}{|l|}{ YFP } \\
\hline \multirow[t]{2}{*}{ OnTI1 } & 1894 & AAAAAGCAGGCTTCATGGAGTTCATCAAAGCAAAG \\
\hline & 1895 & AGAAAGCTGGGTCTTAAGGAGTACACTITTATC \\
\hline \multirow[t]{2}{*}{ OnTI2 } & 1892 & AAAAAGCAGGCTTCATGGAGCTCATCAAATCATCA \\
\hline & 1893 & AGAAAGCTGGGTCTTAATTACTAGTACATTTATT \\
\hline \multirow[t]{2}{*}{ OnTI3 } & 2124 & AAAAAGCAGGCTTCATGGAGTTCATCAAA \\
\hline & 2125 & AGAAAGCTGGGTCTTAAGGAGTACACTGTT \\
\hline \multirow[t]{2}{*}{ OnExpansin } & 1898 & AAAAAGCAGGCTTCATGACACCATCCCTCTTCCTC \\
\hline & 1899 & AGAAAGCTGGGTCTCAAAACTGCGCGCCCTCGAA \\
\hline \multirow[t]{2}{*}{ OnDRRP } & 1896 & AAAAAGCAGGCTTCATGGCTTCCTTCTCCTTCTCT \\
\hline & 1897 & AGAAAGCTGGGTCCTAATTGTTGTTAAAGACAAC \\
\hline
\end{tabular}

$\mathrm{nm}$ for chloroplast autofluorescence. The images are presented as 3D maxima intensity projected stacks processed with LSM 510 version 4.2 (Zeiss).

\section{Promoter study}

Promoters were amplified using PCR incorporating Onc. GR genomic DNA as the template. Primer information is given in Table 5. Using Gateway Technology (Invitrogen), products were cloned into pDONR221 using Gateway BP Clonase II Enzyme Mix (Invitrogen) and cloned into the binary vector, pHGWFS7 vector, which has a GFP-GUS reporter gene [44], using Gateway LR Clonase II Enzyme Mix (Invitrogen). These plasmids were cotransformed with a reference control, pJD301, containing the luciferase gene driven by the CaMV 35 S promoter [45] into the Onc. GR lips and leaves using bombardment transformation. For histochemistry, bombarded tissues were transferred to a reagent containing $0.1 \mathrm{M}$ phosphate buffer ( $\mathrm{pH} 7.0$ ), $0.5 \mathrm{mM}$ potassium ferricyanide, $0.5 \mathrm{mM}$ potassium ferrocyanide, $0.1 \%$ Triton X-100, $10 \mathrm{mM}$ EDTA, 20\% (v/v) methanol and $1 \mathrm{mM}$ 5-bromo-3-indolyl-glucuronide (Sigma). The tissues were incubated overnight at $37^{\circ} \mathrm{C}$ and cleared using $70 \%$ $(\mathrm{v} / \mathrm{v})$ ethanol. 
Table 5 Primers used in the promoter study

\begin{tabular}{|c|c|c|c|}
\hline Gene & Prom. size & Forward primer & Reverse primer \\
\hline \multirow[t]{5}{*}{ OnTI1 } & 1913 & AAAAAGCAGGCTGTCGACAAAGCCCAATTCATTCCAGT & AGAAAGCTGGGTCATCTAAAGTGATTGTGAGGA \\
\hline & 1340 & AAAAAGCAGGCTTTCATGTTAACAACCATC & AGAAAGCTGGGTCATCTAAAGTGATTGTGAGGA \\
\hline & 920 & AAAAAGCAGGCTCAACTTCATTTACTGTAGCTC & AGAAAGCTGGGTCATCTAAAGTGATTGTGAGGA \\
\hline & 740 & AAAAAGCAGGCTTGAAAAATTGTGAG & AGAAAGCTGGGTCATCTAAAGTGATTGTGAGGA \\
\hline & 360 & AAAAAGCAGGCTCGGAACTCCACAAG & AGAAAGCTGGGTCATCTAAAGTGATTGTGAGGA \\
\hline \multirow[t]{6}{*}{ OnExpansin } & 1027 & AAAAAGCAGGCTGCCCCAAATGACACCTTA & AGAAAGCTGGGTCATTGTTAAGAGTTAGAATTTG \\
\hline & 882 & AAAAAGCAGGCTCTCCTATTGCACCCATTTTC & AGAAAGCTGGGTCATTGTTAAGAGTTAGAATTTG \\
\hline & 746 & AAAAAGCAGGCTTGATTCAACCCATTC & AGAAAGCTGGGTCATTGTTAAGAGTTAGAATTTG \\
\hline & 569 & AAAAAGCAGGCTTGCACAGAGGCAAACATATATTT & AGAAAGCTGGGTCATTGTTAAGAGTTAGAATTTG \\
\hline & 334 & AAAAAGCAGGCTCAACGCAAGTTAACC & AGAAAGCTGGGTCATTGTTAAGAGTTAGAATTTG \\
\hline & 133 & AAAAAGCAGGCTCAGCATGTGCACTTCCACCT & AGAAAGCTGGGTCATTGTTAGAGTTAGAATTTG \\
\hline OnTI2 & 1151 & AAAAAGCAGGCTAAACAAGCTTCTCCCCCTTTG & AGAAAGCTGGGTCATCTTAATAATTAGCTTGTTG \\
\hline OnDRRP & 1024 & AAA AAG CAG GCT AGG AAG GAC ACA CAA CTT & AGA AAG CTG GGT CAT TAG AGA GTA GGA GGT \\
\hline
\end{tabular}

To measure luciferase and GUS activities, $0.4 \mathrm{~g}$ of tissue was ground in a mortar after liquid nitrogen treatment. A volume of $1 \mathrm{ml}$ of $1 \times$ CCLR Buffer (Promega, Madison, WI, USA) was added to the powder and incubated at room temperature for $5 \mathrm{~min}$. The solution was centrifuged at $18,000 \times \mathrm{g}$ for $5 \mathrm{~min}$ and the supernatant collected for further measurements. Luciferase activity was determined using luciferase assay reagent (Promega). GUS-specific activities were determined using $2 \mathrm{mM}$ of 4-methylumbelliferone glucoronide substrate [14].

Transcription binding sites and tandem repeats were analyzed using the Plant Promoter Analysis Navigator [46].

\section{Acknowledgements}

We thank Dr. Yu-Yun Chang for helpful discussions and Dr. Heiko Kuhn and Ms. Miranda Loney for editing the manuscript. This work was supported by the Development Program of Industrialization for Agricultural Biotechnology (to MTC and CSL), the Taiwan Seed Improvement and Propagation Station (to CSL) and Academia Sinica (to MTC and CSL) Taiwan.

\section{Author details}

${ }^{1}$ Agricultural Biotechnology Research Center, Academia Sinica, Taipei, Taiwan. ${ }^{2}$ Scientific Instrument Center, Academia Sinica, Taipei, Taiwan. ${ }^{3}$ Institute of Plant and Microbial Biology, Academia Sinica, Taipei, Taiwan. ${ }^{4}$ Institute of Molecular Biology, Academia Sinica, Taipei, Taiwan. ${ }^{5}$ Institute of Biotechnology, National Chung Hsing University, Taichung, Taiwan.

${ }^{6}$ Academia Sinica Biotechnology Center in Southern Taiwan, Tainan, Taiwan.

\section{Authors' contributions}

$\mathrm{CTH}$ carried out the majority of the experiments, including plasmid constructions and transient transformations. DCL performed the BAC library screening experiments. FHW, NTL, SJC, and SYT carried out the microarray experiments. SCS participated in the microscopy experiments. CHY participated in the Oncidium MADS gene studies. MTC and CSL designed and coordinated experiments. CSL wrote the manuscript. All the authors read and approved the final manuscript.

Received: 28 November 2010 Accepted: 7 April 2011 Published: 7 April 2011

\section{References}

1. Webster P: Oncidium subtribe. The Orchid Genus Book 1992.

2. Zelenko H, Chase MW: Orchids: The Pictorial Encyclopedia of Oncidium. New York: ZAl Publications; Rev., 22002.
3. Lu HC, Chen HH, Tsai WC, Chen WH, Su HJ, Chang DC, Yeh HH: Strategies for functional validation of genes involved in reproductive stages of orchids. Plant Physiol 2007, 143(2):558-569.

4. Liau CH, You SJ, Prasad V, Hsiao HH, Lu JC, Yang NS, Chan MT: Agrobacterium tumefaciens-mediated transformation of an Oncidium orchid. Plant Cell Rep 2003, 21(10):993-998.

5. Hsu HF, Yang CH: An orchid (Oncidium Gower Ramsey) AP3-like MADS gene regulates floral formation and initiation. Plant Cell Physiol 2002, 43(10):1198-1209.

6. Hsu HF, Huang $\mathrm{CH}$, Chou LT, Yang CH: Ectopic expression of an orchid (Oncidium Gower Ramsey) AGL6-like gene promotes flowering by activating flowering time genes in Arabidopsis thaliana. Plant Cell Physiol 2003, 44(8):783-794.

7. Chang YY, Chiu YF, Wu JW, Yang CH: Four orchid (Oncidium Gower Ramsey) AP1/AGL9-like MADS box genes show novel expression patterns and cause different effects on floral transition and formation in Arabidopsis thaliana. Plant Cell Physiol 2009, 50(8):1425-1438.

8. Hou CJ, Yang CH: Functional analysis of FT and TFL1 orthologs from orchid (Oncidium Gower Ramsey) that regulate the vegetative to reproductive transition. Plant Cell Physiol 2009, 50(8):1544-1557.

9. Thiruvengadam M, Yang CH: Ectopic expression of two MADS box genes from orchid (Oncidium Gower Ramsey) and lily (Lilium longiflorum) alters flower transition and formation in Eustoma grandiflorum. Plant Cell Rep 2009, 28(10):1463-1473.

10. Hsu HF, Hsieh WP, Chen MK, Chang YY, Yang CH: C/D class MADS box genes from two monocots, orchid (Oncidium Gower Ramsey) and lily (Lilium longiflorum), exhibit different effects on floral transition and formation in Arabidopsis thaliana. Plant Cell Physiol 2010, 51(6):1029-1045.

11. Wu FH, Chan MT, Liao DC, Hsu CT, Lee YW, Daniell H, Duvall MR, Lin CS: Complete chloroplast genome of Oncidium Gower Ramsey and evaluation of molecular markers for identification and breeding in Oncidiinae. BMC Plant Biol 2010, 10:68.

12. Shen $\mathrm{CH}$, Yeh $\mathrm{KW}$ : Hydrogen peroxide mediates the expression of ascorbate-related genes in response to methanol stimulation in Oncidium. J Plant Physiol 2010, 167(5):400-407.

13. Shen $\mathrm{CH}$, Krishnamurthy $\mathrm{R}$, Yeh KW: Decreased L-ascorbate content mediating bolting is mainly regulated by the galacturonate pathway in Oncidium. Plant Cell Physiol 2009, 50(5):935-946.

14. Chiou CY, Wu K, Yeh KW: Characterization and promoter activity of chromoplast specific carotenoid associated gene (CHRC) from Oncidium Gower Ramsey. Biotechnol Lett 2008, 30(10):1861-1866.

15. Wang CY, Chiou CY, Wang HL, Krishnamurthy R, Venkatagiri S, Tan J, Yeh KW: Carbohydrate mobilization and gene regulatory profile in the pseudobulb of Oncidium orchid during the flowering process. Planta 2008, 227(5):1063-1077.

16. Chiou CY, Yeh KW: Differential expression of MYB gene (OgMYB1) determines color patterning in floral tissue of Oncidium Gower Ramsey. Plant Mol Biol 2008, 66(4):379-388. 
17. Wang HL, Yeh KW, Chen PR, Chang CH, Chen JM, Khoo KH: Isolation and characterization of a pure mannan from Oncidium (cv. Gower Ramsey) current pseudobulb during initial inflorescence development. BiosCi Biotechnol Biochem 2006, 70(2):551-553.

18. Tan J, Wang HL, Yeh KW: Analysis of organ-specific, expressed genes in Oncidium orchid by subtractive expressed sequence tags library. Biotechnol Lett 2005, 27(19):1517-1528.

19. Febrer M, Wilhelm E, Al-Kaff N, Wright J, Powell W, Bevan MW, Boulton Ml Rapid identification of the three homoeologues of the wheat dwarfing gene Rht using a novel PCR-based screen of three-dimensional BAC pools. Genome 2009, 52(12):993-1000.

20. Yim YS, Moak P, Sanchez-Villeda H, Musket TA, Close P, Klein PE, Mullet JE, McMullen MD, Fang Z, Schaeffer ML, et al: A BAC pooling strategy combined with PCR-based screenings in a large, highly repetitive genome enables integration of the maize genetic and physical maps. BMC Genomics 2007, 8:47.

21. Bouzidi MF, Franchel J, Tao Q, Stormo K, Mraz A, Nicolas P, Mouzeyar S: A sunflower BAC library suitable for PCR screening and physical mapping of targeted genomic regions. Theor App/ Genet 2006, 113(1):81-89.

22. Cenci A, Somma S, Chantret N, Dubcovsky J, Blanco A: PCR identification of durum wheat BAC clones containing genes coding for carotenoid biosynthesis enzymes and their chromosome localization. Genome 2004, 47(5):911-917.

23. Nelson BK, Cai $X$, Nebenfuhr A: A multicolored set of in vivo organelle markers for co-localization studies in Arabidopsis and other plants. Plant J 2007, 51(6):1126-1136.

24. Lee LY, Fang MJ, Kuang LY, Gelvin SB: Vectors for multi-color bimolecular fluorescence complementation to investigate protein-protein interactions in living plant cells. Plant Methods 2008, 4:24.

25. Mondragon-Palomino M, Theissen G: MADS about the evolution of orchid flowers. Trends Plant Sci 2008, 13(2):51-59.

26. Barakat A, Bagniewska-Zadworna A, Choi A, Plakkat U, DiLoreto DS, Yellanki P, Carlson JE: The cinnamyl alcohol dehydrogenase gene family in Populus: phylogeny, organization, and expression. BMC Plant Biol 2009, 9:26.

27. Wilkins O, Nahal H, Foong J, Provart NJ, Campbell MM: Expansion and diversification of the Populus R2R3-MYB family of transcription factors. Plant Physiol 2009, 149(2):981-993.

28. Hu R, Qi G, Kong Y, Kong D, Gao Q, Zhou G: Comprehensive analysis of NAC domain transcription factor gene family in Populus trichocarpa. BMC Plant Biol 2010, 10:145.

29. Briesemeister S, Rahnenfuhrer J, Kohlbacher O: Going from where to whyinterpretable prediction of protein subcellular localization. Bioinformatics 2010, 26(9):1232-1238.

30. Wu FH, Shen SC, Lee LY, Lee SH, Chan MT, Lin CS: Tape-Arabidopsis Sandwich - a simpler Arabidopsis protoplast isolation method. Plant Methods 2009, 5:16.

31. Yoo SD, Cho YH, Sheen J: Arabidopsis mesophyll protoplasts: a versatile cell system for transient gene expression analysis. Nat Protoc 2007, 2(7):1565-1572.

32. Galasso I, Piergiovanni AR, Lioi L, Campion B, Bollini R, Sparvoli F: Genome organization of Bowman-Birk inhibitor in common bean (Phaseolus vulgaris L.). Molecular Breeding 2009, 23(4):617-624.

33. Downing WL, Mauxion F, Fauvarque MO, Reviron MP, Devienne D, Vartanian N, Giraudat J: A Brassica napus transcript encoding a protein related to the Kunitz Protease Inhibitor family accumulates upon waterstress in leaves, not in seeds. Plant J 1992, 2(5):685-693.

34. Srinivasan T, Kumar KR, Kirti PB: Constitutive expression of a trypsin protease inhibitor confers multiple stress tolerance in transgenic tobacco. Plant Cell Physiol 2009, 50(3):541-553.

35. Huang $Y$, Xiao $B$, Xiong $L$ : Characterization of a stress responsive proteinase inhibitor gene with positive effect in improving drought resistance in rice. Planta 2007, 226(1):73-85

36. Horisberger $M$, Tacchini-Vonlanthen M: Ultrastructural localization of Bowman-Birk inhibitor on thin sections of Glycine max (soybean) cv. Maple Arrow by the gold method. Histochemistry 1983, 77(3):313-321.

37. Shitan N, Horiuchi K, Sato F, Yazaki K: Bowman-birk proteinase inhibitor confers heavy metal and multiple drug tolerance in yeast. Plant Cell Physiol 2007, 48(1):193-197.

38. Sampedro J, Cosgrove DJ: The expansin superfamily. Genome Biology 2005, 6(12):242.
39. Lee $Y$, Choi D: Biochemical properties and localization of the betaexpansin OsEXPB3 in rice (Oryza sativa L.). Mol Cells 2005, 20(1):119-126.

40. Balestrini R, Cosgrove DJ, Bonfante P: Differential location of alphaexpansin proteins during the accommodation of root cells to an arbuscular mycorrhizal fungus. Planta 2005, 220(6):889-899.

41. Wu FH, Liu NT, Chou SJ, Shen SC, Chang BCH, Pan CY, Lin CS: Identification of repressed gene transcript accumulation in three albino mutants of Bambusa edulis Munro by cDNA microarray analysis. J Sci Food Agr 2009, 89(13):2308-2316.

42. Zhang $Y$, Zhang $X$, Scheuring CF, Zhang H-B, Huan $P$, Li F, Xiang J: Construction and characterization of two bacterial artificial chromosome libraries of Zhikong scallop, Chlamys farreri Jones et Preston, and identification of BAC Clones containing the genes involved in its innate immune system. Mar Biotechnol 2008, 10(4):358-365.

43. Murashige T, Skoog F: A revised medium for rapid growth and bio assays with tobacco tissue cultures. Physiol Plantarum 1962, 15(3):473.

44. Karimi M, Inze D, Depicker A: GATEWAY vectors for Agrobacteriummediated plant transformation. Trends Plant Sci 2002, 7(5):193-195.

45. Luehrsen KR, de Wet JR, Walbot V: Transient expression analysis in plants using firefly luciferase reporter gene. Methods Enzymol 1992, 216:397-414.

46. Chang WC, Lee TY, Huang HD, Huang HY, Pan RL: PlantPAN: Plant promoter analysis navigator, for identifying combinatorial cis-regulatory elements with distance constraint in plant gene groups. BMCGenomics 2008, 9:561.

doi:10.1186/1471-2229-11-60

Cite this article as: Hsu et al:: Integration of molecular biology tools for identifying promoters and genes abundantly expressed in flowers of Oncidium Gower Ramsey. BMC Plant Biology 2011 11:60.

\section{Submit your next manuscript to BioMed Central and take full advantage of:}

- Convenient online submission

- Thorough peer review

- No space constraints or color figure charges

- Immediate publication on acceptance

- Inclusion in PubMed, CAS, Scopus and Google Scholar

- Research which is freely available for redistribution

Submit your manuscript at www.biomedcentral.com/submit
C Biomed Central 